\title{
Robust square-wave polarization switching in vertical-cavity surface-emitting lasers
}

\author{
M. Marconi, ${ }^{1}$ J. Javaloyes, ${ }^{2}$ S. Barland,${ }^{1}$ M. Giudici, ${ }^{1}$ and S. Balle ${ }^{3}$ \\ ${ }^{1}$ Institut Non Linéaire de Nice, Université de Nice Sophia Antipolis-Centre National de la Recherche Scientifique, 1361 route des lucioles, \\ F-06560 Valbonne, France \\ ${ }^{2}$ Departament de Fisica, Universitat de les Illes Baleares, C/Valldemossa, km 7.5, E-07122 Palma de Mallorca, Spain \\ ${ }^{3}$ Institut Mediterrani d'Estudis Avançats, CSIC-UIB, C/Miquel Marqués, 21, E-07190 Esporles, Spain
}

(Received 1 August 2012; published 23 January 2013)

\begin{abstract}
We study theoretically and experimentally the combined effects of polarization-selective optical feedback and of crossed-polarization reinjection in a vertical-cavity surface-emitting laser. We show that the application of polarization-selective optical feedback, which induces an effective dichroism in the system, allows one to generate a robust and regular square-wave output signal in each polarization component. The period of the square-wave signal is determined by twice the reinjection delay. We analyze the regularity of the induced modulation as a function of laser bias current, dichroism, and of the levels and delays of reinjection and feedback, thus revealing the robustness of the square-wave emission in the parameter space. We also show that the feedback is more effective when acting on the long-wavelength polarization mode, which can be traced to the asymmetry of the bistable region of the vertical-cavity surface-emitting lasers for low dichroism.
\end{abstract}

DOI: 10.1103/PhysRevA.87.013827

PACS number(s): 42.55.Px, 42.60.Lh

\section{INTRODUCTION}

The possibility of inducing emission of regular or chaotic signals in semiconductor lasers under steady conditions of bias current has attracted a lot of interest in the past two decades [1]. In fact, while semiconductor lasers offer many advantages as photonic sources, signal generation via modulation of the injection current requires high-speed electronics and is limited in bandwidth due to the electrical characteristics of the laser diode and its mounting. Alternative solutions have been proposed where the laser emission is governed by a dynamical regime induced by either the presence of optical feedback [2,3], coupling to other lasers [4], optical injection $[5,6]$, or polarization-rotated optical feedback $[7,8]$. The advantage of these solutions is that they are selfsustained and robust, provided that their stability range is sufficiently extended in the parameter space of the system considered. Moreover, bandwidth limitations of the output signal come from the internal time scales of the laser (intraband and/or interband relaxation processes, photon lifetime, etc.) rather than from the electrical parasitics in the laser package.

In particular, vertical cavity surface emitting lasers (VCSELs) have been proposed as promising devices for implementing useful dynamics taking advantage of their polarization degree of freedom [9]. VCSELs present many advantages: single longitudinal and transverse (when the transverse size is properly sized) mode emission, circular output beam, and low cost of fabrication. However, even single-longitudinal and single-transverse mode VCSELs exhibit instabilities due to the absence of a strong polarization anisotropy capable of fixing the polarization emission. Often, single-mode VCSELs show polarization switching when the injection current is scanned. This was initially attributed to the temperature rise in the VCSEL cavity due to joule heating, which redshifts the gain spectrum at a higher rate than the polarization modes [10]. Later experiments [11-13] evidenced that more complicated mechanisms may lead to polarization switching, which required more refined models [14-16].
When VCSELs are subject to optical feedback, the polarization stability is affected and polarization dynamics appear even in the case of perfectly isotropic feedback [17]. Polarizationrotated optical feedback - where the two linearly polarized components, LP-x and LP-y, of the light emitted by the device are fed back into the laser cavity after the LP-x component is converted into the LP-y component and vice versa-induces a regular polarization dynamics which can be as fast as $9 \mathrm{GHz}[7,8]$.

Another scheme which has been investigated for generating optical pulses is based on crossed-polarization re-injection (XPR) both in edge-emitting devices [18] and VCSELs $[19,20]$. In such a scheme, the emission from the laser is split into its two linearly polarized components, LP-x and LP-y, and only one of these components (LP-x, say) is fed back into the laser after being rotated into the orthogonal polarization direction (LP-y). In this way, cross saturation of the polarization states modifies the photon density in each LP component and may eventually lead to pulsed emission with a fundamental time scale given by the reinjection delay $\tau_{r}$. When the dichroism in the device is high enough and the emission occurs on a single polarization mode, strong enough XPR leads to square-wave modulation of the power emitted in each of the LP components with a period close to $2 \tau_{r}$. According to the analysis in [18], the square-wave regime corresponds to a period-two solution on the slow time scale $\tau_{r}$ that describes a quasistatic orbit between two distinct stages, which appear as intensity plateaus in the polarization-resolved time trace. The former stage (first plateau) corresponds to emission of the only stable polarization in the solitary system, and the latter (second plateau) to amplification of the reinjected light in the orthogonal polarization. Both stages last for a time $\tau_{r}$ that corresponds to the time of flight in the reinjection arm.

In VCSELs, the range of currents where XPR is effective for generating square-wave modulation of the output is limited. In VCSELs that have low dichroism-hence which may display bistability in some parameter range- the square-wave modulation progressively degrades as the parameters approach the bistable regime and the regularity of the signal is quite 
sensitive on the working point and strongly affected by small deviations from the optimal parameter values [19]. An explanation of this degradation mechanism based on the bifurcation diagram of the VCSEL submitted to XPR was detailed in [19] where it was shown that the second plateau of the square wave was affected by the vicinity of the LP-y state and by the unstable limit cycle around it, hereby distorting the regularity of the square wave form. On the other hand, when the dichroism is large, square-wave signals can be obtained over large ranges of bias currents, although it was reported in [20] that the quality of the square-wave signal degrades as the current is increased.

In this paper we demonstrate, both experimentally and theoretically, that is is possible to extend the current range of square-wave modulation of a VCSEL having a low dichroism by submitting it to both XPR and weak polarization-selective optical feedback (PSF). A fraction of the output of one polarization component (LP-X, say) is reflected at an external mirror and fed back into the VCSEL after a delay $\tau_{f}$; in addition, another fraction of the same polarization component is rotated to the orthogonal polarization direction (LP-y) and reinjected into the VCSEL after a delay $\tau_{r}$. The presence of PSF decreases the losses of the selected polarization, and can be seen as an external mechanism for controlling the effective dichroism in the device. As such, PSF can effectively bring the device into a monostable regime, hereby reinforcing the effectiveness of XPR for the generation of a very regular square-wave emission which turns out to be very robust versus parameter changes. Interestingly, the impact of PSF in such a process is different for the different LP components, as evidenced both theoretically and experimentally. The proposed scheme allows one to generate square-wave signals from VCSELs with weak dichroism where XPR alone would not work, and it largely increases the range of parameters where stable and regular signals are obtained.

\section{THEORY}

\section{A. Model}

In order to theoretically analyze the behavior of the VCSEL and compare with the experimental results, we use the so-called spin-flip model (SFM) [14], suitably modified for incorporating the effects of both PSF and XPR. Although the natural basis for the SFM is that of the circularly polarized components of the field, $E_{ \pm}=(X \pm i Y) / \sqrt{2}$, the simplest way to incorporate PSF and XPR is in terms of the linearly polarized components of the field, $(X, Y)$. In this linearly polarized base, the model reads

$$
\begin{aligned}
\dot{X}= & (1+i \alpha)[(N-1) X+i n Y]-\left(\gamma_{a}+i \gamma_{p}\right) X \\
& +\eta e^{-i \Omega}\left[a X\left(t-\tau_{f}\right)+b Y\left(t-\tau_{r}\right)\right], \\
\dot{Y}= & (1+i \alpha)[(N-1) Y-i n X]+\left(\gamma_{a}+i \gamma_{p}\right) Y, \\
& +\beta e^{-i \theta}\left[a X\left(t-\tau_{r}\right)+b Y\left(t-\tau_{f}\right)\right], \\
T \dot{N}=\mu & -N\left(1+|X|^{2}+|Y|^{2}\right)-i n\left(X^{\star} Y-X Y^{\star}\right), \\
T \dot{n}=- & n\left(\gamma_{s}+|X|^{2}+|Y|^{2}\right)-i N\left(X^{\star} Y-X Y^{\star}\right),
\end{aligned}
$$

where $N(n)$ is the scaled total (difference of) carrier density in the two spin channels. The terms $X\left(t-\tau_{f, r}\right)$ and $Y\left(t-\tau_{f, r}\right)$ in the evolution equations for the $X$ and $Y$ components describe the effects of PSF and of XPR, which have strengths $\eta$ and $\beta$, respectively, with time delays $\tau_{f}$ and $\tau_{r}$, respectively. $a$ and $b$ describe which LP component experiences feedback and reinjection: when LP-x experiences PSF and is reinjected turned into LP-y, then $a=1$ and $b=0$, and vice versa.

In Eqs. (1)-(4), time has been scaled to the cavity decay rate $\kappa$, while $T=\kappa / \gamma_{e}$ represents the scaled carrier lifetime and $\gamma_{s} / T$ is the decay rate of the spin difference. The rate of carrier density injected into the active region due to the bias current is represented by $\mu$. In addition, $\alpha$ stands for the linewidth enhancement factor [21] and $\gamma_{a}\left(\gamma_{p}\right)$ describes the linear dichroism (birefringence) of the VCSEL cavity. Finally, we have added to the time evolution independent Langevin sources $\left(F_{x}(t), F_{y}(t), F_{N}(t), F_{n}(t)\right)$, describing noise due to spontaneous emission and current fluctuations, whose properties are described in Ref. [22]. The spontaneous emission factor amplitude is controlled by the scaled variance parameter $\eta_{s p}$, that we take as $\eta_{s p}=10^{-4}$. We assume standard values for Henry's factor, $\alpha=2$, a normalized carrier lifetime $T=150$, and a typical normalized spin-flip rate $\gamma_{s}=75$. Assuming that $\kappa=300 \mathrm{~ns}^{-1}, T=150$ and $\gamma_{s}=75$ correspond to a carrier lifetime of $0.2 \mathrm{~ns}$ and a spin-difference decay time of $6.6 \mathrm{ps}$, respectively.

The polarization switchings in the SFM have been exhaustively analyzed in the literature [15,23]; in this model, the VCSEL displays large ranges of bistability when dichroism and birefringence are small [15]. We choose $\gamma_{p}=+5.24 \times$ $10^{-2}$, which means that we denote by LP-x the reddest mode. For this ensemble of parameters, the stability diagram of the solitary VCSEL as a function of the bias current and of the dichroism is depicted in Fig. 1. For small $\left|\gamma_{a}\right|$, the range of

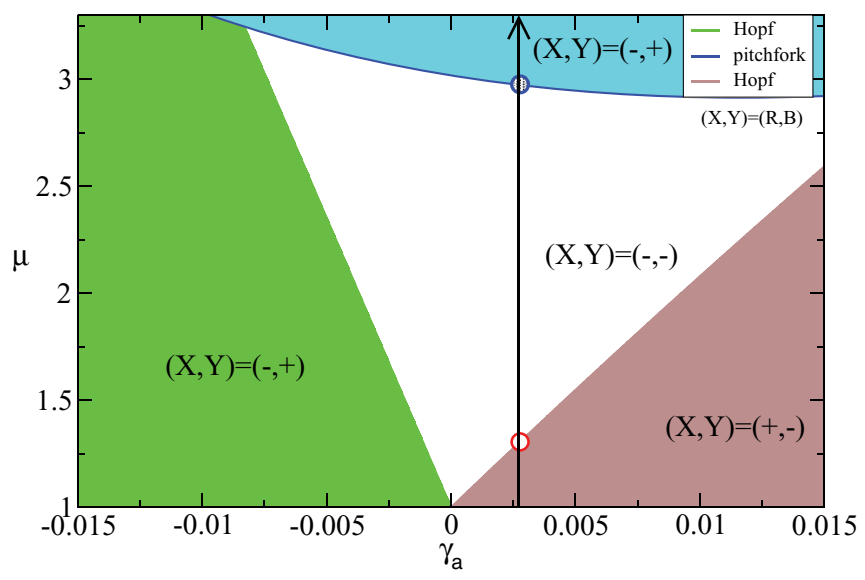

FIG. 1. (Color online) Stability diagram of the solitary VCSEL for $\gamma_{p}>0$, i.e., the LP-y mode being the bluest. Modal stability is indicated as $(X, Y)=(a, b)$, where a negative value denotes stable and vice versa. The vertical line depicts a current scan in the bistability diagram. In the case shown (LP-y mode selected at threshold), the red (blue) circle at low (high) value of $\mu$ represents the critical value of the current $\mu_{h}\left(\mu_{\epsilon}\right)$, where the LP-x (LP-y) mode gains (loses) stability via a subcritical Hopf (supercritical pitchfork) bifurcation; see [23] for details. 
bistability extends over large intervals of bias current. For instance, when $\gamma_{a}=2.6 \times 10^{-3}$, the VCSEL is monostable on the blue polarization from $\mu_{t h}^{Y}=1-\gamma_{a}$ to $\mu_{h} \sim 1.3$ and then bistable until $\mu_{\epsilon} \sim 2.9$; similarly, for $\gamma_{a}=-2.6 \times 10^{-3}$, the red polarization is only stable between $\mu_{t h}^{X}=1+\gamma_{a}$ and $\mu_{h} \sim 1.7$ and then bistable until $\mu_{\epsilon} \sim 3.1$. Note, however, that in practice this range can be reduced due to joule heating as current is increased: the subsequent redshift of the gain curve relative to the mode spectrum favors the reddest mode [10]. Since we are mainly interested in analyzing the effects of PSF and XPR on the dynamics in the bistable regime, we disregard any changes in dichroism as current is varied.

\section{B. Numerical results}

In this subsection we present results for the numerical simulation of the extended SFM in Eqs. (1)-(4), which, as will be discussed later, are in excellent agreement with the experimental results. If not otherwise stated, the PSF and the XPR rates are chosen to be real, i.e., $\Omega=\theta=0$, while the delays are $\tau_{r}=900$ and $\tau_{f}=2 \tau_{r}$, which correspond to $6 \mathrm{~ns}$ and $12 \mathrm{~ns}$, respectively, if a standard cavity decay rate $\kappa=300 \mathrm{~ns}^{-1}$ is considered. For definiteness, we take $\gamma_{a}=2.6 \times 10^{-3}$ and $(a, b)=(0,1)$, i.e., the LP-y component is selected for PSF and used for XPR.

We show in Fig. 2 both a short segment of the time traces and the power spectra of the LP-y component of the VCSEL under the action of XPR $(\beta=0.3)$ as the level of PSF is increased. There is a critical value of the feedback rate $\eta_{c} \simeq$ 0.01 below which no dynamics can be observed. The existence of a minimum PSF level $\eta_{c}$ for developing the square-wave signal can be understood as the level of feedback required to drive the system, via the effective dichroism due to PSF, into the monostable parameter regimes. Above $\eta_{c}$, a square-wave signal develops and its quality improves with the feedback level for some range, but eventually it degrades since too large values of $\eta$ allow many modes of the external cavity to compete

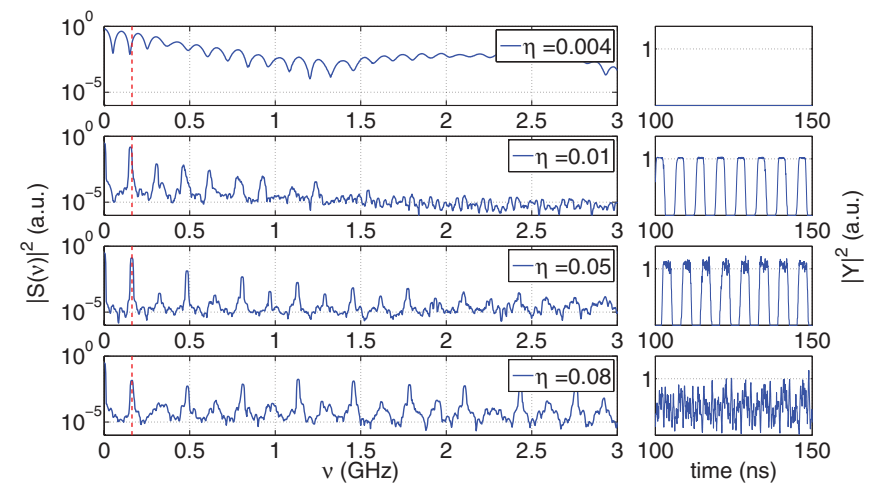

FIG. 2. (Color online) Power spectra (left) and temporal traces (right) of the intensity of the LP-y components of the VCSEL for increasing values of the PSF rate $\eta$ with $\mu=2$ and $\beta=0.3$. For too small values of $\eta$, the system operates on the stable LP-x polarization, and there is an optimal feedback value $\eta \sim 0.01$ above which the regularity of the square waves deteriorates. The vertical red line in the spectra marks the frequency spacing of the external cavity modes.

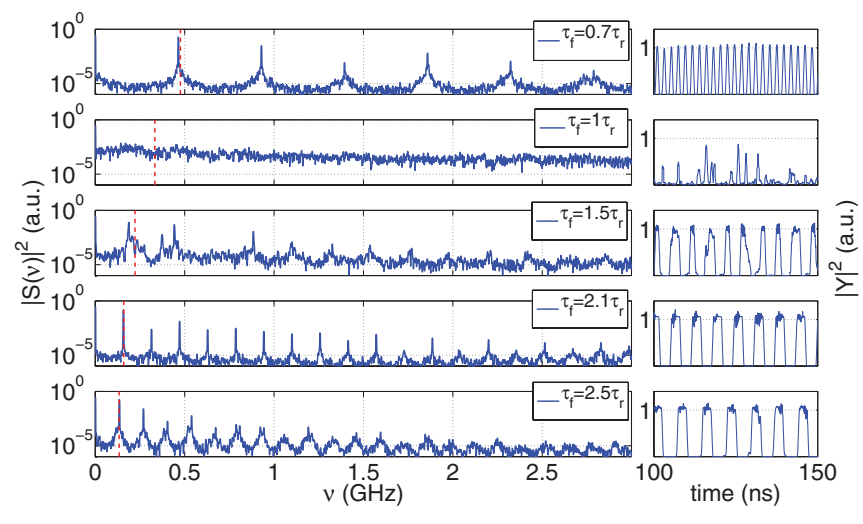

FIG. 3. (Color online) Power spectra (left panel) and traces (right panel) of the intensity of the LP-y component of the VCSEL for increasing values of the PSF delay $\tau_{f}$, with $\eta=0.03, \mu=2$, and $\beta=0.3$. The vertical red line in the spectra marks the spacing of the external cavity modes.

for the gain, hereby creating a complex oscillation instead of a clean plateau.

The optimal value of the PSF rate has to be defined with respect to a somewhat arbitrary criterion. If we consider the suppression of the even harmonics and the similarity of the power spectrum to that of an ideal square-wave signal-which has power ratios $\left|a_{k} / a_{0}\right|^{2}=1 / k^{2}$ for the odd harmonics $k=1,3,5, \ldots$-then the optimal value for these working conditions is in between $\eta \sim 0.03$ and $\eta \sim 0.06$.

Within this regime, we did not notice an influence of the feedback phase $\Omega$ on the square-wave dynamics, only on the amount of noise present on the signal. This can be understood from the fact that during the second plateau of the square wave, the LP-y feedback is reinjected into an "off"laser (i.e., LP-x emitting), and the feedback intensity has simply to be sufficient to expel the system from the basin of attraction of the LP-x mode allowing one to restart another cycle of the square wave.

Instead (see Fig. 3) the value of the PSF delay $\tau_{f}$ compared to the XPR delay $\tau_{r}$ has a paramount influence on the dynamics. The analysis of plateaux in [18] indicates that PSF will help in regularizing the square-wave signal if the PSF onto the LP-y arrives close to the end of the second plateau, since this will accelerate the transition from LP-x into LP-y emission, i.e., for PSF delays that verify $\tau_{f} \sim 2 n \tau_{r}$ with $n$ an integer. Indeed, the numerical simulations in Fig. 3 show that goodquality square waves are obtained for $1.5 \tau_{r}<\tau_{f}<2.5 \tau_{r}$. An alternative assessment of the quality of the square-wave signal in this range can be obtained by looking at the power spectrum of the signal and comparing it with that of a perfect square-wave signal, which as discussed before contains only the odd harmonics of the fundamental frequency, with power decreasing as the square of the harmonic index. Indeed, we observe (see Fig. 4) that power of the second harmonic has a minimum and that in third harmonic is about one-tenth of that in the first harmonic for $\tau_{f}=2 \tau_{r}$. Around this region, a good-quality rectangular signal is still obtained, but the duty-cycle progressively deviates from $50 \%$, hence increasing the power in the second harmonic.

For feedback cavities shorter than $\tau_{f} / \tau_{r} \simeq 0.8$, the power in each component exhibits fast oscillations with a dominant 


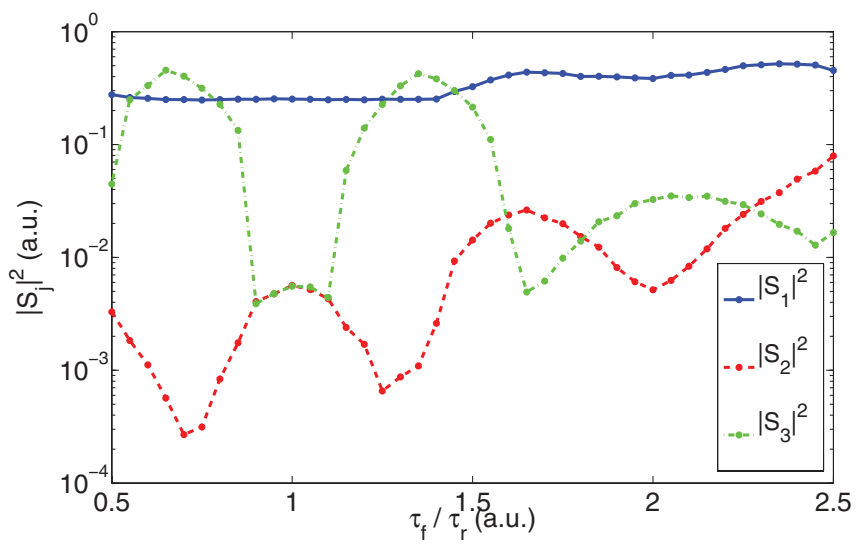

FIG. 4. (Color online) Peak power in the first (solid blue), second (dashed red), and third (dash-dotted green) harmonic of the squarewave signal as a function of the delay in the PSF arm normalized to the XPR delay.

frequency that roughly correspond to the third harmonic of the fundamental frequency of the squares and to the fundamental frequency of the PSF delay. For long cavities, the excitation of multiple external cavity modes destroys the square wave, at least in the range of cavity lengths explored here.

We conclude our analysis by studying the effect of the bias current on the square-wave quality. In the optimal case depicted in Fig. 5, where $\tau_{f}=2 \tau_{r}$, one can see that square waves are present from close to threshold up to three times the threshold. We found that the quality of the square-wave oscillation as inferred from the spectra in Fig. 5 steadily increases with the bias current; see, for instance, Fig. 5 obtained with $\mu=2$. This is at variance with the analysis developed in [19] where it was shown that there is an optimal bias current value above which the square-wave oscillation deteriorates. It was shown in [19] that XPR has the side effect of rendering the Hopf bifurcation of the LP-y mode supercritical instead of subcritical, which has the effect of anticipating bistability (see Fig. 9 of Ref. [19]). As a consequence, when the bias current approaches this regime, the vicinity of the limit cycle distorts the orbit imposed by

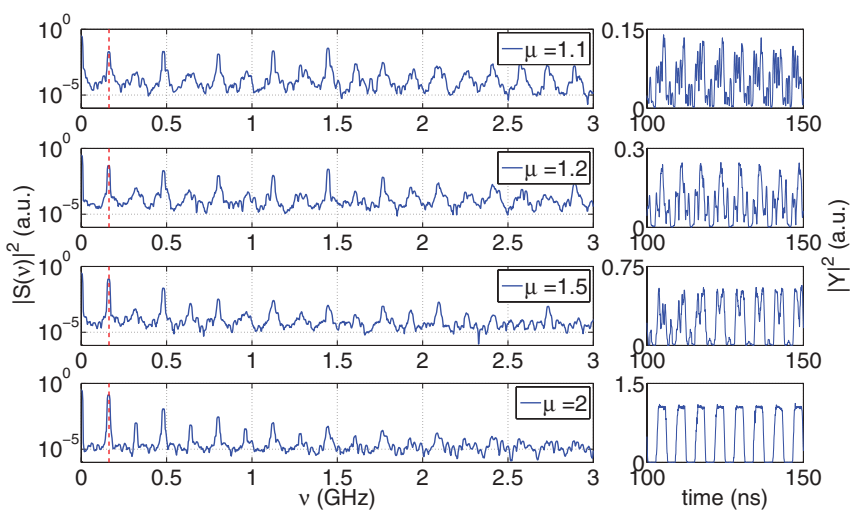

FIG. 5. (Color online) Power spectra (left) and temporal traces (right) of the intensity of the LP-y components of the VCSEL for increasing values of the bias current $\mu$, with $\eta=0.03, \Omega=0, \mu=2$, and $\beta=0.3$ in the optimal case where $\tau_{f}=2 \tau_{r}$. The quality of the square-wave oscillation is strictly increasing with the bias current since no bistability is encountered along the way.

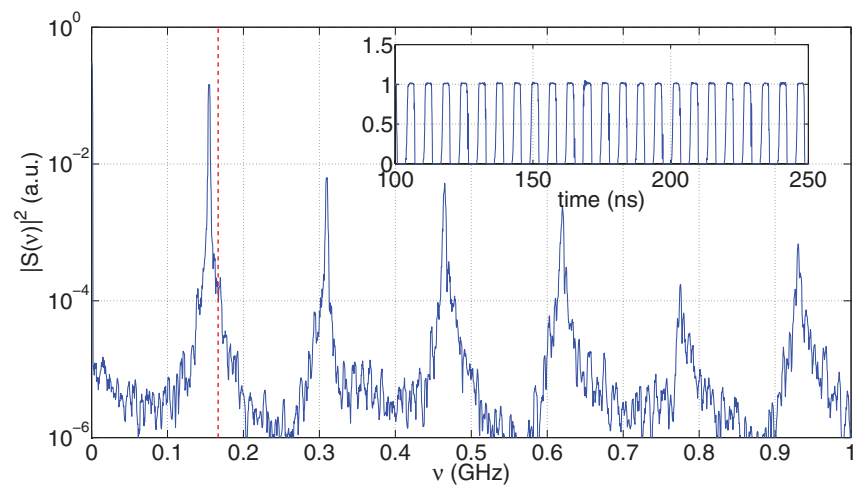

FIG. 6. (Color online) Power spectra and time trace (inset) of the LP-x polarization when the LP-x is fed back and re-injected turned into LP-y. The parameters are $\gamma_{a}=-2.6 \times 10^{-3}, \mu=2$, and $\beta=0.15$. The feedback rate is $\eta=0.005$.

$\mathrm{XPR}$, thus destroying the wave form regularity. It is worth remarking that this deterioration is mitigated here due to the presence of PSF, and that the combination of XPR and PSF allows one to largely increase the parameter space domain (both with respect to the XPR strength and bias current) where the square-signal output exhibits a high degree of regularity.

\section{Asymmetric sensitivity to feedback}

The numerical results in the previous subsection have been obtained in the case where PSF favors the LP-y component, i.e., the mode with the highest optical frequency and the lowest threshold. Qualitatively similar results are obtained when PSF favors LP-x, but it is observed that, in this second case, the levels of PSF needed to achieve good-quality square waves were systematically lower than in the first case.

This remarkable asymmetry between the two cases regarding the quality of the square-wave signal is obtained independent of the value of $\gamma_{a}$, as exemplified in Figs. 6 and 7. For the sake of definiteness, we assume here that $\gamma_{a}=-2.6 \times 10^{-3}$ and $\gamma_{p}=5.24 \times 10^{-2}$, i.e., the solitary VCSEL is dominantly emitting LP-x (the reddest polarization component) and it operates on the left part of the bistable region; see Fig. 1 with $\mu=2$ and $\gamma_{a}=-2.6 \times 10^{-3}$. Due

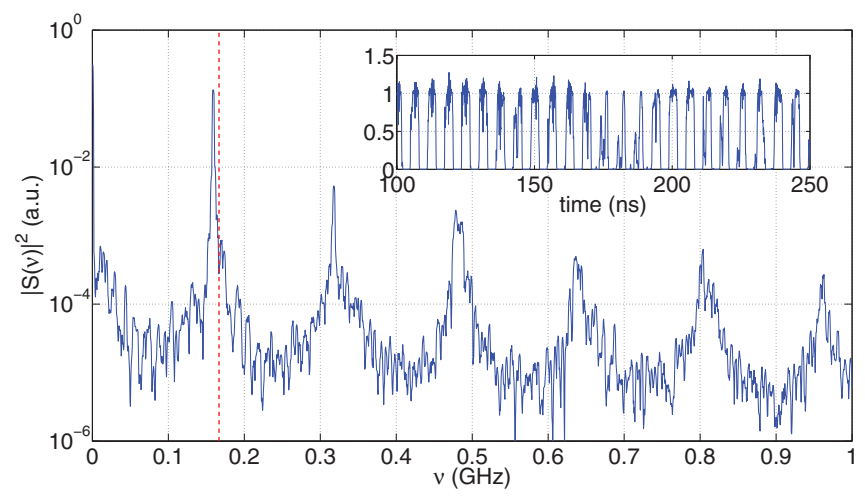

FIG. 7. (Color online) Power spectra and time trace (inset) of the LP-y polarization when the LP-y is fed back and re-injected turned into LP-x. The parameters are $\gamma_{a}=-2.6 \times 10^{-3}, \mu=2$, and $\beta=0.15$. The feedback rate is $\eta=0.02$. 
to the bistability, the square wave cannot be obtained using only XPR. Since dichroism in our devices is rather small, minute quantities of (resonant) feedback should be sufficient to destroy the bistability since the system is close to the region where the red mode (the green area in Fig. 1) is the only one stable. Hence we use $\eta=0.005$, which indeed yields a regular square output, as seen in Fig. 6.

Oppositely, an identical amount of feedback in the bluest polarization component (LP-y) does not allow for square waves as the system enters more deeply into the bistable region. Here, the minimal value needed is close to $\eta=0.02$, which allows reaching the brown region where the LP-y is the only stable mode. One can see in Fig. 7 that the square-signal regularity is clearly worse than in the previous case in spite of all the system parameters being identical except for the polarization component selected for PSF and XPR.

The different effectiveness of PSF for the different LP components can be qualitatively understood when considering the effect of the PSF as a modification of the effective dichroism and making reference to the asymmetry of the bistable region; see Fig. 1. For small values of the dichroism (as the ones considered here), the higher slope of the border on the $\gamma_{a}<0$ side implies that, in general, a lower PSF strength is required to leave the bistability region when selecting LP-x instead than LP-y.

For a given rate of PSF, the system can be driven into a polarization monostable region. If PSF is applied on LP-x, it is pushed deeper into the green left region than if the same amount of PSF is applied on LP-y. In this case, the system is pushed into the brown right region but it lies closer to the bistable region than in the former case. Hence the vicinity of the bistable region leads to a less regular and robust square-wave signal. The higher minimal value of the feedback required in the latter case implies a larger number of active external cavity modes that eventually lead to more complex and irregular dynamics. Conversely, in the case of positive values of dichroism (yet such that the VCSEL is in the bistable range), the situation would be reversed.

\section{EXPERIMENTAL SETUP}

The setup used for characterizing and controlling the solitary VCSEL polarization is described in Fig. 8. The light emitted from a thermally stabilized VCSEL $\left( \pm 0.001{ }^{\circ} \mathrm{C}\right)$ driven with a low-noise current source $( \pm 1 \mu \mathrm{A})$ is collimated and sent to a 50:50 polarization-preserving beam splitter (BS) after passing through a half-wavelength wave plate $(\lambda / 2)$ that allows one to align the VCSEL polarization axes to the horizontal and vertical direction with respect to the optical table.

The BS divides the beam in two, both having the same intensity. The beam deflected by the BS passes through a Glan-Taylor polarizing beam splitter (PBS) and a partially reflecting mirror (FM) which sends the beam back into the VCSEL, thus providing PSF. The PBS is set to transmit the polarization component parallel to the optical table while deflecting the orthogonal one. Thus the optical feedback circuit returns the same polarization after a delay $\tau_{f}=2 L_{\mathrm{PSF}} / c$, $L_{\mathrm{PSF}}$ being the optical path length from the VCSEL to the feedback mirror. The XPR arm is realized by placing a $45^{\circ}$

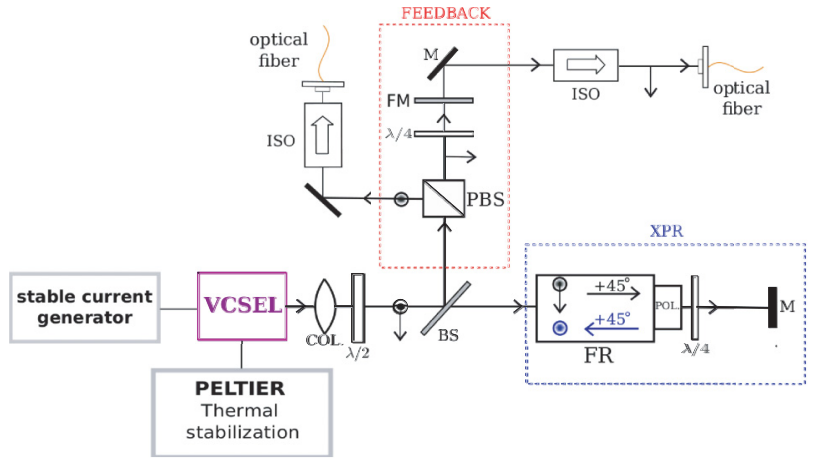

FIG. 8. (Color online) Experimental setup. COL: collimator. $\lambda / 2$ : half-wavelength wave plates. $\lambda / 4$ : quarter-wavelength wave plates. BS: Polarization-preserving beam splitter. PBS: Polarizing beam splitter. FR: Faraday rotator. POL: Polarizer. FM: Feedback mirror. M: Mirror. ISO: Optical isolator.

Faraday rotator with an exit polarizer (i.e., an optical isolator where the input polarizer has been removed) on the path of the beam transmitted by the BS. Upon reflection on a mirror (M), only one of the LP components of the light is reinjected back into the VCSEL, after experiencing a $90^{\circ}$ rotation of its polarization orientation. The Faraday rotator axes with their exit polarizer are oriented such that the LP component that passes through the XPR circuit and which is rotated $90^{\circ}$ before being reinjected into the VCSEL is the same selected by the PSF arm, i.e., the polarization component parallel to the optical table. The delay in the XPR circuit is given by $\tau_{r}=2 L_{\mathrm{XPR}} / c$, $L_{\mathrm{XPR}}$ being the optical path length from the VCSEL to mirror M.

In order to quantify the maximum rates of PSF and XPR, we measure the fraction of the emitted power that is returned back to the VCSEL along the corresponding circuit somewhere between the $\lambda / 2$ placed at the VCSEL output and the polarization preserving BS. These measurements are performed checking that the VCSEL is emitting in a single linear polarization; moreover, in order to avoid interaction of these returned beams with the VCSEL and thus altering the measurement of rates, the returned beams were slightly misaligned with respect to the optical axis. The maximum rates for XPR and PSF achievable in our setup are of $17 \%$ and of $5 \%$, respectively. A quarter-wavelength wave plate $(\lambda / 4)$ placed before each of the mirrors used for closing the PSF and XPR circuits finally allows for independent fine-tuning of the PSF and XPR rates.

Polarization-resolved detection of the VCSEL emission is performed on the feedback circuit by monitoring both the beam transmitted through the semireflecting feedback mirror and the beam deflected by the Glan-Taylor PBS. We use two photodiode detectors ( $8 \mathrm{GHz}$ bandwidth) connected to a $6 \mathrm{GHz}$ digital scope and a $20 \mathrm{GHz}$ power spectrum analyzer.

We have tested two VCSELs from ULM-photonics lasing at $850 \mathrm{~nm}$ (ULM.850-PMTNS46FOP). They are single mode with a suppression ratio larger than $10 \mathrm{~dB}$ at the rated power of $1 \mathrm{~mW}$. Both devices exhibit a birifringence of $7.5 \pm 0.5 \mathrm{GHz}$ and both emit linearly polarized light. Their threshold is around $0.5 \mathrm{~mA}$ with the bluest polarization mode, that we 
call LP-y, appearing at threshold. Both devices may exhibit a polarization switch at a current value $J_{\text {switch }}$ depending on substrate temperature. In the first device, $J_{\text {switch }} \simeq 1.3 \mathrm{~mA}$ at $T=20^{\circ} \mathrm{C}$; however, $J_{\text {switch }}$ decreases as temperature is increased and, at $T=30^{\circ} \mathrm{C}$, it cannot be distinguished from the laser threshold. In the second device, $J_{\text {switch }}$ is much closer to threshold at ambient temperature, and we need to cool down the device to less than $T=10^{\circ} \mathrm{C}$ for clearly distinguishing a polarization switching.

The first device exhibits polarization bistability above $J_{\text {switch }}$ over a current range much larger than what could be expected from the polarization-resolved LI curves, as evidenced by transitorily applying PSF or XPR to the system. For instance, if the VCSEL is emitting on LP-x above $J_{\text {switch }}$, one can apply PSF to the LP-y component or XPR of LP-x light into LP-y. Upon a given rate of PSF the laser emission abruptly switches to LP-y, and it continues to emit on this state after PSF is removed. Similarly, when XPR is applied, the emission abruptly passes to be dominated by LP-y, and when XPR is removed, the VCSEL emits pure LP-y light. These operations are reversible and can be realized in the parameter space for currents between $J_{\text {switch }}$ and the absolute maximum rating $(J=3 \mathrm{~mA})$. In the second device, it is possible to induce the same switch from the two polarization states when applying PSF (or XPR) which also occurs abruptly when the PSF (or XPR) rate exceeds a given value; in this case, however, the VCSEL emission comes back to LP-x a short time after PSF (or XPR) is removed. Thus the two devices show bistable behavior over a large current range, although the second device is only weakly bistable.

\section{EXPERIMENTAL RESULTS}

We focus now on the dynamics obtained when the VCSELs are submitted to XPR and PSF. The results obtained depend on the polarization component which is selected to pass through the feedback circuit and the Faraday rotator. Both VCSELs tested show qualitatively similar results versus parameter changes.

The bistable character of the VCSELs under test does not allow one to obtain a square-wave signal in any configuration when only XPR is acting, as shown in the left column of Fig. 9 for the first device at $T=30^{\circ} \mathrm{C}$. Let us consider the case of LP-y selected by the XPR circuit (top panel). If VCSEL parameters are such that emission is on LP-y, the power in the LP-y decreases (and that in the LP-x component increases) almost linearly as the XPR rate is increased. When the XPR rate is raised above $0.5 \%$, an abrupt increase of the LP-X component and an abrupt decrease in the LP-y component are observed, but the VCSEL is emitting stationarily in a mixed polarization state. If the XPR is further increased, an instability appears in both polarization components, their dynamics being out of phase. At the highest achievable XPR rate, the power spectrum exhibits a first peak at $v=c / 4 L_{\mathrm{XPR}}=\left(2 \tau_{r}\right)^{-1}$ and only odd harmonics of this fundamental frequency are present, as previously observed in [19]. Nevertheless, the peaks are broad and shallow, and indeed the regular square-wave emission observed in [19] could not be observed in these VCSELs for any parameter set: the most regular situation is obtained for $J=0.85 \mathrm{~mA}$, as shown in Fig. 9, upper panel.
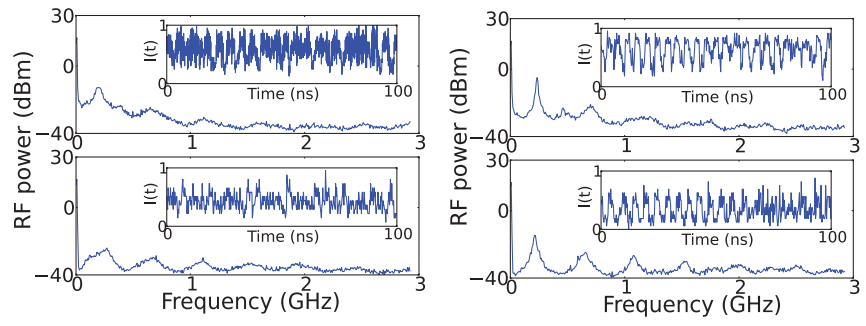

FIG. 9. (Color online) Upper panels: power spectrum and corresponding time signal (inset) of the LP-x output of the first device when $J=0.85 \mathrm{~mA}, T=30^{\circ} \mathrm{C}$, and the VCSEL is submitted to XPR only (rate 17\%) of the LP-y component (left column) and both to XPR (rate $17 \%$ ) and PSF (rate $0.15 \%$ ) on LP-y (right column). Lower panels: power spectrum and corresponding time signal (inset) of the LP-y output when $J=0.55 \mathrm{~mA}$ and the VCSEL is submitted to XPR only (rate 17\%) of the LP-x component (left column) and both to XPR (rate 17\%) and PSF (rate $0.15 \%$ ) on LP-x (right column). In all cases, $L_{\mathrm{XPR}}=33 \mathrm{~cm}$ and $L_{\mathrm{PSF}}=58.8 \mathrm{~cm}$.

If we set XPR selecting LP-X and we set the XPR rate at the highest level, a power spectrum with odd harmonics of the fundamental frequency appears only for currents very close to laser threshold, $J=0.55 \mathrm{~mA}$, but the time trace reveals a poor quality of the square wave form (Fig. 9, lower panel in left column).

Our VCSELs, then, do not produce good-quality squarewave signals by XPR only, which is not surprising considering their bistable behavior [19]; in fact, as discussed in [19], the bistability of the VCSEL is detrimental for the formation of a square-wave signal.

In order to improve the quality and robustness of the squarewave signals generated by the VCSELs, we provide PSF on the same polarization component that is allowed to pass through the XPR circuit. As discussed in Sec. II, PSF is expected to change the effective dichroism between LP states, hereby breaking polarization bistability and helping the development of the square-wave oscillation. Indeed, we observe a qualitative improvement of the regularity of the signal in both cases, either selecting LP-y (Fig. 9, right column, upper panel) or LP-x (Fig. 9, right column, lower panel). In order to explore the existence and robustness of a regular square-wave signal when using both XPR and PSF, we perform an analysis versus the system parameters.

The time behavior of LP-y component when the VCSEL is submitted to both XPR and PSF selecting LP-x is shown in Fig. 10 for different rates of PSF. The length of the external cavity providing PSF is set to $L_{\mathrm{PSF}}=98 \mathrm{~cm}$ and the length of the XPR circuit is set to $L_{\mathrm{XPR}}=49 \mathrm{~cm}$; thus the optical feedback delay corresponds to twice the delay for XPR. The rate of XPR is the maximum $(17 \%)$, and when increasing the feedback level, we observe the sequence shown in Fig. 10.

For a very small feedback rate, the signal is noisy and its power spectrum shows excess noise at the harmonics of the main peak given by $c / 4 L_{\mathrm{XPR}}$. As the feedback level is increased, a regular square-wave signal develops and settles down for a feedback strength of $0.12 \%$. For a feedback level of $0.44 \%$ the regularity is maximized, and its power spectrum displays narrow peaks at the harmonics of the fundamental 


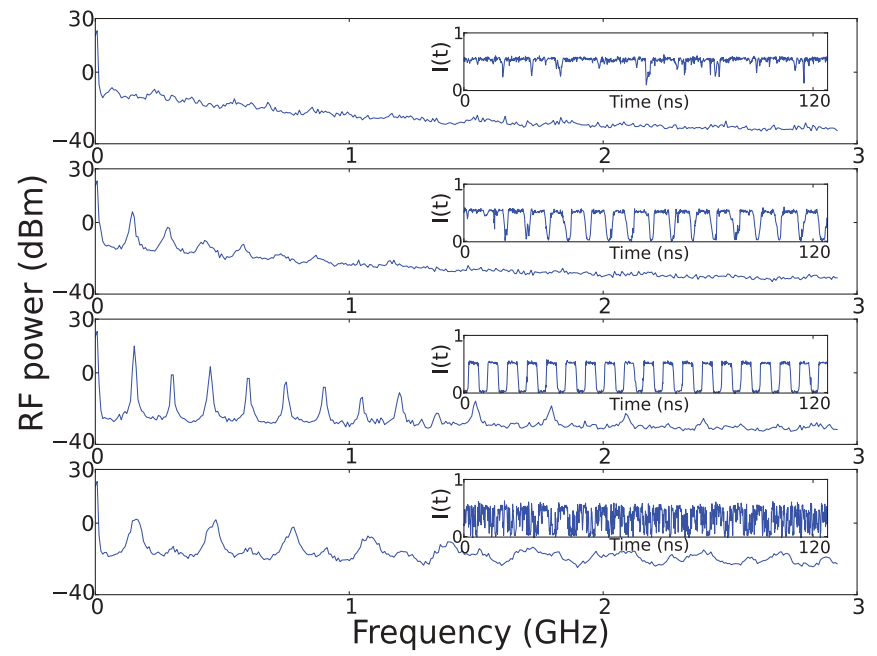

FIG. 10. (Color online) Power spectrum and time trace (inset) of the LP-y signal when the VCSEL is submitted to both XPR and PSF of the LP-x component for $J=3 \mathrm{~mA}, T=30^{\circ} \mathrm{C}, L_{\mathrm{PSF}}=98 \mathrm{~cm}$, and $L_{\mathrm{XPR}}=49 \mathrm{~cm}$. The XPR rate is $17 \%$ and the PSF rates are, from top to bottom, $0.03 \%, 0.05 \%, 0.44 \%$, and $2.5 \%$, respectively.

frequency $v_{r} \simeq c / 4 L_{\mathrm{XPR}}=\left(2 \tau_{r}\right)^{-1} ;$ the odd harmonics clearly dominate the spectrum, the even harmonics being suppressed by more than $10 \mathrm{~dB}$ with respect to the preceding odd harmonics. However, when the PSF level is above $\sim 1 \%$, the output signal becomes irregular again, and the square wave form eventually disappears.

The robustness of this square signal has been assessed also versus pumping current. In Fig. 11 we show how the dynamics evolves when changing $J$. For a wide current range
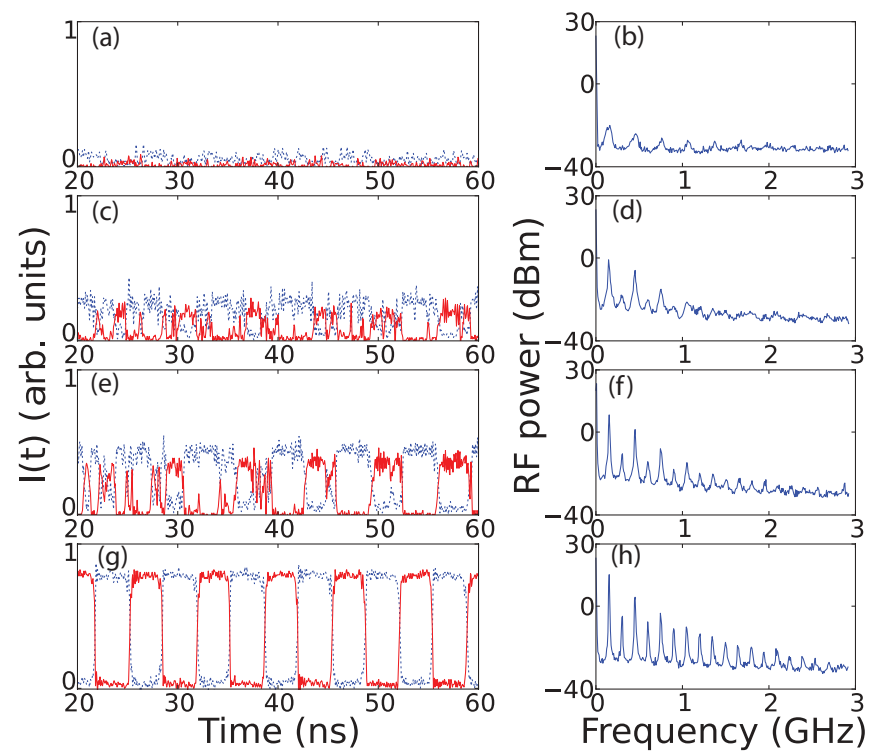

FIG. 11. (Color online) Left column: power of the LP-y (blue dotted trace) and LP-x (red trace) components when the VCSEL is submitted to both XPR and PSF of the LP-x component for $L_{\mathrm{PSF}}=$ $98 \mathrm{~cm}, L_{\mathrm{XPR}}=49 \mathrm{~cm}, T=30^{\circ} \mathrm{C}, \mathrm{PSF}=0.4 \%$, and XPR $=17 \%$ for $J=0.5 \mathrm{~mA}$ (a), $J=1.0 \mathrm{~mA}$ (c), $J=1.5 \mathrm{~mA}$ (e), and $J=$ $3.0 \mathrm{~mA}(\mathrm{~g})$. Right column: corresponding power spectrum of the LP-y component.
$2 \mathrm{~mA}<J<3 \mathrm{~mA}$, the square signal appears regular with the same characteristics shown in the optimal parameter set of Fig. 10. When the current is decreased down to $1 \mathrm{~mA}$ the regularity is lost. Synchronous and balanced acquisition of the two polarization components reveals that, for $2 \mathrm{~mA}<J<$ $3 \mathrm{~mA}$, the square-wave oscillations in the two polarizations are in antiphase and have the same amplitude as can be observed in Fig. 11, thus leading to a constant total intensity output of the VCSEL. In the most regular situation, obtained in Fig. 11 for $J=3 \mathrm{~mA}$, the full width at half maximum of the first peak is of $200 \mathrm{kHz}$, while the suppression of the first even harmonic peak over the fundamental peak is larger than $20 \mathrm{~dB}$.

We remark that the regularity of this square signal is quite sensitive to the XPR rate; in fact, if the rate of XPR is decreased below $16 \%$, the regularity is rapidly lost and a noisy signal is found. This is in agreement with the existence of a minimum threshold for the rate of XPR yielding squares, as discussed in [19]; unfortunately, this value is very close to the maximum level of XPR attainable in our setup.

We have also characterized the square signal versus the ratio of delays, $\tau_{f} / \tau_{r}$. As discussed above, the first peak in the power spectrum and the repetition rate of the square signal are given by $v_{r} \simeq c / 4 L_{\mathrm{XPR}}=\left(2 \tau_{r}\right)^{-1}$. If $\mathrm{XPR}$ is removed, the power spectrum exhibits peaks at integer multiples of $v_{f}=c / 2 L_{\mathrm{PSF}}=\tau_{f}{ }^{-1}$, the inverse of the round-trip time in the external feedback cavity.

Figure 12 shows that the existence of the square signal depends on the ratio of the delays. For an XPR delay of $\tau_{r}=2.6 \mathrm{~ns}$ and varying $\tau_{f}$ by steps of $200 \mathrm{ps}$ we have observed that the square signal appears for $3.4 \mathrm{~ns}<\tau_{f}<5.4 \mathrm{~ns}$, which
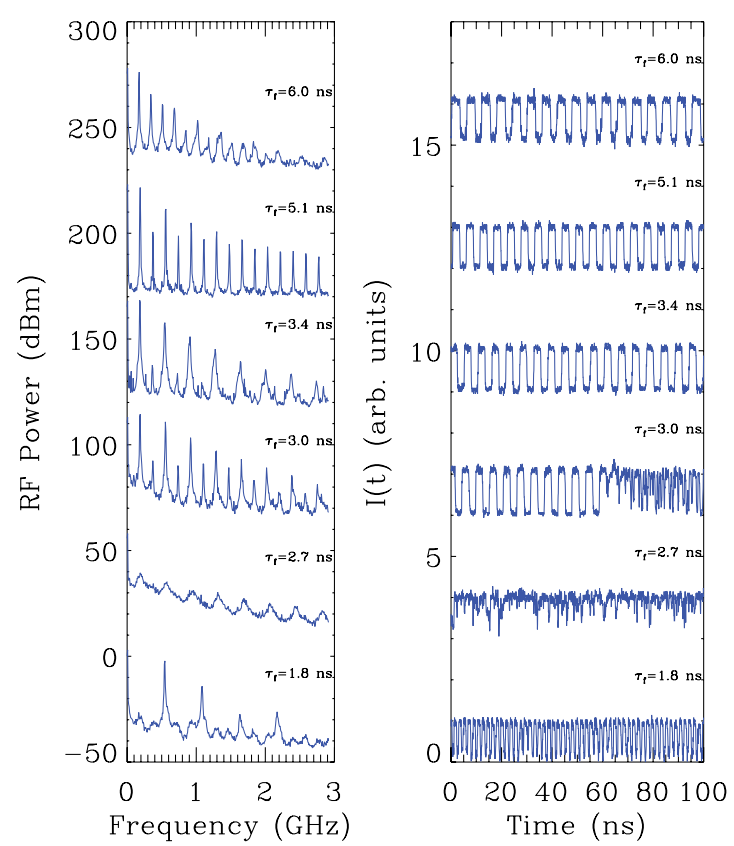

FIG. 12. (Color online) Power spectra (left panel) and temporal signal (right panel) of the LP-y polarization when the VCSEL is submitted to both XPR (rate 17\%) and PSF (rate 0.1\%) of the LP-x component, for different values of $\tau_{f}$ as indicated. $\tau_{r}=2.6 \mathrm{~ns}$ and $J=2.5 \mathrm{~mA} ; T=30^{\circ} \mathrm{C}$. For clarity, the spectra have been vertically offset by $55 \mathrm{~dB}$ and the time traces have been shifted by 3 units. 

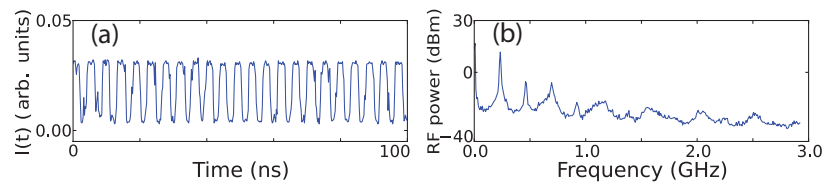

FIG. 13. (Color online) LP-x output emission together with LP-x power spectrum when the VCSEL is submitted to both XPR and polarization-selective optical feedback. Both XPR and optical feedback circuits are set to select LP-y and all parameters are optimized to obtain the most regular square signal. $L_{\text {feed }}=58.8 \mathrm{~cm}$, $L_{\mathrm{XPR}}=69.8 \mathrm{~cm}, \mathrm{PSOF}=0.15 \%, \mathrm{XPR}=17 \%, J=2.8 \mathrm{~mA}$, and $T=30^{\circ} \mathrm{C}$.

in terms of delay ratio means $1.3<\frac{\tau_{f}}{\tau_{r}}<2.1$. In this range, the regularity of the square signal is maximum in the range $1.7<\frac{\tau_{f}}{\tau_{x_{F}}}<2.1$, and the square wave is not sensitive to the feedback phase. The only effect of the feedback phase in the range of cavity lengths explored is to modify the noise on the square-wave signal, increasing by a few $\mathrm{dB}$ the noise floor level in the square-wave power spectrum.

When $\tau_{f}$ is decreased below this range, the regularity is progressively lost; bursts of irregular behavior appear for $\tau_{f}=3 \mathrm{~ns}$ and get more and more dominant in time as $\tau_{f}$ is decreased, until, for $\tau_{f}=2,7 \mathrm{~ns}\left(\frac{\tau_{f}}{\tau_{r}} \approx 1\right)$, the signal is completely irregular. Further decreasing $\tau_{f}$ below $\frac{\tau_{f}}{\tau_{r}} \approx 0.7$ leads to an oscillating signal whose power spectrum exhibits only peaks at integer multiples of the third harmonic of $v_{r}$ (Fig. 12 for $\tau_{f}=1.8 \mathrm{~ns}$ ). When $\tau_{f}$ is further decreased the signal becomes again very irregular and its power spectrum shows excess noise at several frequencies that can be associated to multiples of $v_{r}$ and multiples of $v_{f}$. Instead, for $\tau_{f}>$ $6 \mathrm{~ns}$ (i.e., for $\frac{\tau_{f}}{\tau_{r_{r}}}>2.3$ ) the regularity is rapidly degraded and the peaks in the power spectra broaden showing a substructure with smaller peaks that can be associated to $v_{f}$ and multiples.

The results presented up to now correspond to the case where LP-x polarization is selected for PSF and XPR. When LP-y is selected for feedback and reinjection, qualitatively similar phenomena are obtained. In this case, however, although the presence of PSF increases significantly the regularity of the output signal obtained with XPR only, we were unable to observe a square-wave signal as regular as the one observed when selecting LP-x. In Fig. 13 we show the most regular signal we were able to obtain selecting LP-y. This signal was obtained by optimizing the delay times and levels of XPR and PSF, as well as the laser pumping current. The square-wave modulation obtained by using LP-y for XPR and PSF is stable in a much narrower parameter region than when using LP-x. The square-wave signal is now stable only over $0.5 \mathrm{~mA}$ in pump current and, in terms of delays ratio, there is only a sharp interval where the regularity is preserved.

\section{CONCLUSIONS}

We have studied theoretically and experimentally the generation of square-wave signals from bistable VCSELs submitted both to XPR and to weak PSF. The presence of PSF decreases the losses of the selected polarization providing a mechanism which effectively increases the dichroism and that reinforces the effectiveness of XPR for the generation of a very regular square-wave emission which is very robust versus parameter changes. For high enough feedback levels, the VCSEL is effectively driven into a regime where only one polarization state can exist, and the square-wave signal develops. Interestingly, the impact of PSF in such a process is different for the different LP components, as evidenced both theoretically and experimentally, due to the asymmetry of the bistability region with respect to dichroism. The proposed scheme allows one to generate square-wave signals from VCSELs with weak dichroism where XPR alone would not work.

\section{ACKNOWLEDGMENTS}

S.B. acknowledges financial support from project TEC2009-14581-C02-1. J.J. acknowledges financial support from Ministerio de Ciencia e Innovación (Spain), subprograma Ramón y Cajal. S.B. and J.J. acknowledge financial support from the Direcció General de Recerca, Desenvolupament Tecnològic i Innovació de la Conselleria d'Innovació, Interior i Justícia del Govern de les Illes Balears cofunded by the European Union FEDER funds.
[1] D.-L. Cheng and T.-C. Yen, Opt. Commun. 271, 503 (2007).

[2] A. Tager and K. Petermann, IEEE J. Quantum Electron. 30, 1553 (1994).

[3] T. Erneux, A. Gavrielides, and M. Sciamanna, Phys. Rev. A 66, 033809 (2002).

[4] D. W. Sukow, A. Gavrielides, T. Erneux, B. Mooneyham, K. Lee, J. McKay, and J. Davis, Phys. Rev. E 81, 025206 (2010).

[5] E.-K. Lee, H.-S. Pang, J.-D. Park, and H. Lee, Phys. Rev. A 47, 736 (1993).

[6] I. Gatare, M. Sciamanna, M. Nizette, H. Thienpont, and K. Panajotov, Phys. Rev. E 80, 026218 (2009).

[7] F. Robert, P. Besnard, M. Chares, and G. Stephan, IEEE J. Quantum Electron. 33, 2231 (1997).

[8] H. Li, A. Hohl, A. Gavrielides, H. Hou, and K. D. Choquette, Appl. Phys. Lett. 72, 2355 (1998).
[9] K. Panajotov, M. Sciamanna, I. Gatare, M. Arteaga, and H. Thienpont, Adv. Opt. Technol. 2011, 469627 (2011).

[10] K. Choquette, R. Schneider, K. Lear, and R. Leibenguth, IEEE J. Sel. Top. Quantum Electron. 1, 661 (1995).

[11] J. Martín-Regalado, J. L. Chilla, J. J. Rocca, and P. Brusenbach, Appl. Phys. Lett. 70, 3350 (1997).

[12] T. Ackemann and M. Sondermann, Appl. Phys. Lett. 78, 3574 (2001).

[13] M. Sondermann, M. Weinkath, T. Ackemann, J. Mulet, and S. Balle, Phys. Rev. A 68, 033822 (2003).

[14] M. San Miguel, Q. Feng, and J. V. Moloney, Phys. Rev. A 52, 1728 (1995).

[15] J. Martín-Regalado, F. Prati, M. San Miguel, and N. Abraham, IEEE J. Quantum Electron. 33, 765 (1997).

[16] S. Balle, E. Tolkachova, M. San Miguel, J. Tredicce, J. MartínRegalado, and A. Gahl, Optics Letters 24, 1121 (1999). 
[17] M. Giudici, T. Ackemann, S. Barland, J. Tredicce, and S. Balle, J. Opt. Soc. Am. B 16, 2114 (1999).

[18] A. Gavrielides, T. Erneux, D. W. Sukow, G. Burner, T. McLachlan, J. Miller, and J. Amonette, Opt. Lett. 31, 2006 (2006).

[19] J. Mulet, M. Giudici, J. Javaloyes, and S. Balle, Phys. Rev. A 76, 043801 (2007).
[20] D. W. Sukow, T. Gilfillan, B. Pope, M. S. Torre, A. Gavrielides, and C. Masoller, Phys. Rev. A 86, 033818 (2012).

[21] C. Henry, IEEE J. Quantum Electron. 18, 259 (1982).

[22] J. Mulet, C. R. Mirasso, and M. San Miguel, Phys. Rev. A 64, 023817 (2001).

[23] T. Erneux, J. Danckaert, K. Panajotov, and I. Veretennicoff, Phys. Rev. A 59, 4660 (1999). 